\title{
Generalized Solutions to Conservation Laws
}

\author{
M. Oberguggenberger and Y.-G. Wang
}

\begin{abstract}
In this paper we study generalized solutions in the sense of Colombeau to the Cauchy problem for hyperbolic conservation laws and their parabolic approximations. We obtain existence and uniqueness results both in the scaler case and for systems. The relation of the generalized solution to the classical solution, when the latter exists, is established. An application of our results is to the p-system of gas dynamics with artificial viscosity.
\end{abstract}

Key words: Hyperbolic conservation laws, parabolic approximation, genenalized solutions AMS Subject Classiffeation: 35K55, 35L65, 35D05, 46L10, 76N 15

\section{Introduction}

This article is devoted to the study of generalized solutions to hyperbolic conservation laws in one space dimension

$$
\begin{cases}u_{t}+f(u)_{x}=0 & \text { for } x \in \mathbb{R}, t>0 \\ \left.u\right|_{t=0}=u_{0}(x) & \text { for } x \in \mathbb{R}\end{cases}
$$

and the corresponding parabolic approximation

$$
\begin{cases}u_{t}+f(u)_{x}=\mu u_{x x} & \text { for } x \in \mathbb{R}, t>0 \\ \left.q u\right|_{t=0}=u_{0}(x) . & \text { for } x \in \mathbb{R}\end{cases}
$$

with $\mu>0$. We study both the scalar case, $u: \overline{\mathbb{R}_{+}^{2}}=\mathbb{R} \times[0, \infty) \longrightarrow \mathbb{R}$, and the case of a system $u: \overline{\mathbb{R}_{+}^{2}} \longrightarrow \mathbb{R}^{n}$. We are concerned with the construction of solutions in the algebra $\mathcal{G}_{g}\left(\mathbb{R}_{+}^{2}\right)$ of generalized functions, a modified version of the algebras introduced by Colombeau $[4,5]$ to deal with the multiplication problem for distributions. We note that $\mathcal{G}_{g}(\mathbb{R})$ contains the space of bounded distributions $\mathcal{D}_{L^{\infty}}(\mathbb{R})$, so that highly singular initial data can be considered in this setting.

This article is a continuation of the study in [2], where the Burgers equation was investigated, i.e. $f(u)=\frac{1}{2} u^{2}$. It was observed there that (1.1) cannot have solutions with equality in $\mathcal{G}_{g}\left(\mathbb{R}_{+}^{2}\right)$ in the shape of shock waves, but should be replaced by

$$
\left\{\begin{array}{l}
u_{t}+f(u)_{x} \approx 0 \\
\left.u\right|_{t=0}=u_{0}(x)
\end{array}\right.
$$

where $\approx$ denotes the association relation on $\mathcal{G}_{g}\left(\mathbb{R}_{+}^{2}\right)$. On the other hand, the Burgers

M. Oberguggenberger: Univ. Innsbruck, Inst. Math. und Geom., Technikerstr: 13, A-6020 Innsibruek Y.-G. Wang: Shanghai Jiao Tong Univ., Appl. Math., 200030 Shanghai, P.R. China

ISSN 0232-2064 / \$2.50 C Heldermann Verlag Berlin 
equation with viscosity coefficient $\mu>0$ was shown to have unique solutions in $\mathcal{G}_{g}\left(\mathbb{R}_{+}^{2}\right)$. It was demonstrated that shock wave solutions to $(1.1)$, with $f(u)=\frac{1}{2} u^{2}$, could be properly modelled by means of generalized solutions to (1.2) with an infinitesimal generalized coefficient $\mu \approx 0$. These solutions were seen to actually satisfy (1.3) and to behave (in the sense of association) just as the weak entropic $L^{\infty}$-solutions to (1.1), if the initial data belonged to $L^{\infty}(\mathbb{R})$. For a discussion of various aspects of this approach we refer to the introduction of [2].

In this article we extend these results to problems (1.1) and (1.2) with general flux function $f$, only required to be smooth and polynomially bounded, together with all derivatives. Both in the case of a scalar equation and a system we show that there exists a unique generalized solution of (1.2) for arbitrary data in $\mathcal{G}_{g}(I R)$ when $f^{\prime}$ is globally bounded. In the case of a system this remains true when $f^{\prime}$ is globally bounded on an invariant region for (1.2) which contains the initial data. For scalar equations, we'get existence and uniqueness also for unbounded $f^{\prime}$, but a link between the growth of $f^{\prime}$ and the type of the initial data is required (this becomes only relevant when the initial data are singular; no restriction appears when $\left.u_{0} \in L^{\infty}(I R)\right)$.

Further, in the scalar case we show that when $u_{0}$ is a classical bounded function and $\mu \approx 0$, the generalized solution to (1.2) is associated with the weak entropic $L^{\infty}$-solution to the conservation law (1.1). What concerns systems, our results can be applied, for example, to the $p$-system of gas dynamics with artificial viscosity

$$
\left\{\begin{array}{l}
v_{t}-u_{x}=\mu v_{x x}, u_{t}+p(v)_{x}=\mu u_{x x} \\
\left.(v, u)\right|_{t=0}=\left(v_{0}(x), u_{0}(x)\right)
\end{array}\right.
$$

with $p(v)=k v^{-\gamma}, k>0, \gamma>1$.

The plan of exposition is as follows. In Section 2 we recall the definitions from the theory of generalized functions needed in the sequel and introduce some supplementary notions. In Section 3 we state and prove the existence-uniqueness results in the scalar case, and we study the associated distribution when the initial data are classical $L^{\infty}$. functions. Section 4 contains the extension to systems.

\section{Algebras of generalized functions}

In the sequel, we let $\mathbb{R}_{+}^{2}=\mathbb{R} \times(0, \infty)$ and $\overline{R_{+}^{2}}=\mathbb{R} \times[0, \infty)$. The algebra of smooth functions on $\mathbb{R}$ (resp. $\mathbb{R}_{+}^{2}$ ), all whose derivatives are bounded, will be denoted by $\mathcal{C}_{b}^{\infty}(\mathbb{R})$ (resp. $\mathcal{C}_{b}^{\infty}\left(I_{+}^{2}\right)$ ). Further, we let

$$
\mathcal{C}_{b}^{\infty}\left(\mathbb{R}_{+}^{2}\right)=\left\{u \in \mathcal{C}^{\infty}\left(\mathbb{R}_{+}^{2}\right)|u|_{R \times(0, T)} \in \mathcal{C}_{b}^{\infty}(\mathbb{R} \times(0, T)) \text { for all } T>0\right\}
$$

We remark that each element of $\mathcal{C}_{b}^{\infty}\left(\mathbb{R}_{+}^{2}\right)$ has a smooth extension up to $\{t=0\}$, so that $\mathcal{C}_{b}^{\infty}\left(\mathbb{R}_{+}^{2}\right)=\mathcal{C}_{b}^{\infty}\left(\overline{\mathbb{I R}_{+}^{2}}\right)$, similarly for $\mathcal{C}_{\bar{b}}^{\infty}$.

For simplicity, we introduce only the two algebras of generalized functions $\mathcal{G}_{g}\left(\mathbb{R}_{+}^{2}\right)$ and $\mathcal{G}_{g}(I R)$ which we are interested in; for the general definitions we refer to Biagioni [1: Chapter 1], Colombeau ([4: Section 3.4] and [5: Chapter 1]) as well as [8: Sections 9 and 12]. We denote by $\mathcal{E}_{g}\left[\mathbb{R}_{+}^{2}\right]$ the set of all maps $u:(0, \infty) \times \mathbb{R}_{+}^{2} \longrightarrow \mathbb{R}$ such that

$$
(x, t) \longrightarrow u(\epsilon, x, t) \in \mathcal{C}_{b}^{\infty}\left(\mathbb{R}_{+}^{2}\right) \quad \text { for all } \epsilon>0 .
$$


Next, $\mathcal{E}_{M, g}\left[\mathbb{R}_{+}^{2}\right]$ comprises those elements $u$ of $\mathcal{E}_{g}\left[\mathbb{R}_{+}^{2}\right]$ that, for all $(\alpha, \beta) \in \mathbb{N}^{2}$ and $T>0$, there is $N \in \mathbb{N}$ with

$$
\sup _{(x, t) \in R \times(0, T)}\left|\partial_{x}^{\alpha} \partial_{\imath}^{\rho} u(\epsilon, x, t)\right|=O\left(\epsilon^{-N}\right) \quad \text { as } \epsilon \longrightarrow 0 .
$$

Finally, $\mathcal{N}_{g}\left(\mathbb{R}_{+}^{2}\right)$ is the subset of all $u \in \mathcal{E}_{g}\left[\mathbb{R}_{+}^{2}\right]$ such that, for all $(\alpha, \beta) \in \mathbb{N}^{2}, q \in \mathbb{N}$ and $T>0$, it holds

$$
\sup _{(x, t) \in \mathbb{R} \times(0, T)}\left|\partial_{x}^{\alpha} \partial_{i}^{\beta} u(\epsilon, x, t)\right|=O\left(\epsilon^{q}\right) \quad \text { as } \epsilon \longrightarrow 0 .
$$

We note that $\mathcal{E}_{M, g}\left[\mathbb{R}_{+}^{2}\right]$ is a differential algebra, the operations defined componentwise at fixed $\epsilon$, and $\mathcal{N}_{g}\left(\mathbb{R}_{+}^{2}\right)$ is a differential ideal.

Definition 2.1: The differential algebra $\mathcal{G}_{g}\left(\mathbb{R}_{+}^{2}\right)$ is the factor algebra $\mathcal{G}_{g}\left(\mathbb{R}_{+}^{2}\right)=$ $\mathcal{E}_{M, g}\left(\mathbb{R}_{+}^{2}\right) / \mathcal{N}_{g}\left(\mathbb{R}_{+}^{2}\right)$.

The representatives of an element $u \in \mathcal{G}_{g}\left(\mathbb{R}_{+}^{2}\right)$ will be denoted by the symbol $\left\{u_{e}\right\}_{e>0}$ or $\left\{u_{c}(x, t)\right\}_{\curlywedge}>0$. The one-dimensional version $\mathcal{G}_{g}(I R)$ is defined in complete analogy, based on

$$
\mathcal{E}_{g}[R]=\left\{u:(0, \infty) \times \mathbb{R} \longrightarrow \mathbb{R} \text { such that } x \longrightarrow u(\epsilon, x) \in \mathcal{C}_{b}^{\infty}(\mathbb{R}) \text { for all } \epsilon>0\right\} .
$$

We have noted above that $\mathcal{C}_{\delta}^{\infty}\left(\mathbb{R}_{+}^{2}\right)=\mathcal{C}_{\bar{b}}^{\infty}\left(\overline{R_{+}^{2}}\right)$. Accordingly, we can define the restriction of a generalized function to $\{t=0\}$ as follows.

Definition 2.2: Given $u \in \mathcal{G}_{g}\left(\mathbb{R}_{+}^{2}\right)$, its restriction $\left.u\right|_{t=0} \in \mathcal{G}_{g}(\mathbb{R})$ is defined as the class of $\left\{u_{c}(x, 0)\right\}_{c>0}$, where $\left\{u_{c}(x, t)\right\}_{c>0}$ is a representative of $u$.

Concerning nonlinear functions of elements of the algebra $\mathcal{G}_{\rho}\left(\mathbb{R}_{+}^{2}\right)$, we introduce the following notion.

Definition 2.3: A smooth function $f: \mathbb{R}^{n} \longrightarrow \mathbb{R}^{n}$. is called slowly increasing at infinity, if for all $\alpha \in \mathbb{N}^{n}$ there are $N_{\alpha} \in \mathbb{N}$ and $C_{\alpha}>0$ such that $\left|f^{(\alpha)}(u)\right| \leq C_{\alpha}(1+|u|)^{N_{0}}$ for all $u \in \mathbb{R}^{n}$. The number $N_{0}$ is called the order of $f$.

If $f$ is slowly increasing and $u \in\left(\mathcal{G}_{g}\left(\mathbb{R}_{+}^{2}\right)\right)^{n}$ we can define $f(u) \in\left(\mathcal{G}_{g}\left(\mathbb{R}_{+}^{2}\right)\right)^{n}$ on representatives. Thus all operations involved in systems (1.i) or (1.2) have a well-defined meaning in the algebra $\mathcal{G}_{g}\left(\mathbb{R}_{+}^{2}\right)$, given $u_{0} \in \mathcal{G}_{g}(\mathbb{R})$.

We now specify an imbedding of $\mathcal{D}_{L^{\infty}}(\mathbb{R})$ into $\mathcal{G}_{g}(\mathbb{R})$. Fix an element $\rho \in \mathcal{S}(\mathbb{R})$, a rapidly decreasing smooth function with

$$
\int \rho(x) d x=1 \quad \text { and } \quad \int x^{\alpha} \rho(x) d x=0 \text { for all } \alpha \in \mathrm{N}
$$

and set $\rho_{\mathrm{c}}(x)=\epsilon^{-1} \rho\left(\epsilon^{-1} x\right)$ for $x \in \mathbb{R}$ and $\epsilon>0$. The map

$$
\iota_{\rho}: w \longrightarrow \text { class of }\left\{w * \rho_{c}\right\}_{c>0}
$$

defines an imbedding of $\mathcal{D}_{L^{\infty}}(\mathbb{R})$ into $\mathcal{G}_{g}(\mathbb{R})$. It is obvious that $\iota_{\rho}$ commutes with derivation. Further, if $w$ happens to belong to $\mathcal{C}_{b}^{\infty}(I R)$, it may itself serve as a representative 
for $\iota_{\rho}(w)$, as follows easily from (2.1) and Taylor expansion. Thus $\iota_{\rho}$ turns $\mathcal{C}_{b}^{\infty}(\mathbb{R})$ into a subalgebra of $\mathcal{G}_{g}(\mathbb{R})$.

Definition 2.4: An element $u \in \mathcal{G}_{g}\left(\mathbb{R}_{+}^{2}\right)$ is said to be associated with a distribution $w \in \mathcal{D}^{\prime}\left(\mathbb{R}_{+}^{2}\right)$ if for some (and hence every) representative $\left\{u_{e}\right\}_{e}>0$ of $u$ it holds that $u_{\epsilon} \longrightarrow w$ in $\mathcal{D}^{\prime}\left(\mathbb{R}_{+}^{2}\right)$ as $\epsilon \longrightarrow 0$. We denote this by $u \approx w$.

This concept says the following: If $u$ bas an associated distribution $w$, then it behaves like $w$ when regarded on the level of information of distribution theory (on which approximations with the same weak limit are identified).

Definition 2.5: An element $\mu \in \mathcal{G}_{g}(I R)$ is called a generalized constant if it has a representative $\left\{\mu_{c}\right\}_{c}>0$ which is a constant for each $\epsilon>0$. In case $\mu_{c}$ is also bounded away from zero, more precisely there is $N \in \mathbb{N}$ such that $\epsilon^{N} \leq \mu_{e} \leq \epsilon^{-N}$ for all sufficiently small $\epsilon>0$, we shall call $\mu$ a generalized positive number.

If $\mu$ is a generalized positive number, then so is $1 / \mu$. Cleariy, all positive real numbers are also generalized positive numbers. Further, $\mu$ is associated with zero, $\mu \approx 0$, if and only if $\mu_{\mathrm{c}} \longrightarrow 0$ as $\epsilon \longrightarrow 0$.

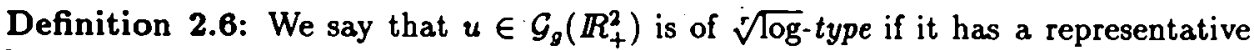
$\left\{u_{c}\right\}_{c>0}$ such that, for every $T>0$,

$$
\sup _{(x, t) \in \mathbb{R} \times(0, T)}\left|u_{e}(x, t)\right|=O(\sqrt[r]{|\log \epsilon|}) \quad \text { as } \epsilon \stackrel{\rightarrow}{\longrightarrow}
$$

It is called of bounded type if

$$
\sup _{(x, t) \in \mathbb{R} \times(0, T)}\left|u_{e}(x, t)\right|=O(1) \quad \text { as } \epsilon \longrightarrow 0 .
$$

A similar definition applies to elements of $\mathcal{G}_{0}(I R)$. We note that if $u_{0} \in L^{\infty}(I R)$, then $\iota_{\rho}\left(u_{0}\right)$ is of bounded type. On the other hand, taking $\rho$ as in $(2.1)$, the map $(\epsilon, x) \stackrel{\because}{\longrightarrow}$ $\sqrt[v]{|\log \epsilon|} \rho(\sqrt[r]{|\log \epsilon|} x)$ is of $\sqrt[r]{\log }$-type and defines an element of $\mathcal{G}_{g}(\mathbb{R})$ associated with the Dirac measure.

The motivation for Definition 2.6 is the validity of the following result, which follows immediately from the definitions.

Lemima 2.7: If $f \in \mathcal{C}^{\infty}(I R)$ is slowly increasing at infinity of order $r$, and $u \in \mathcal{G}_{g}\left(\mathbb{R}_{+}^{2}\right)$ is of $\sqrt[r]{\log -t y p e, ~ t h e n ~} \exp f(u) \in \mathcal{G}_{o}\left(\mathbb{R}_{+}^{2}\right)$. More precisely,

$$
\sup _{(x, t) \in R \times(0, T)} \exp \left|f\left(u_{e}(x, t)\right)\right|=O(1 / \epsilon)
$$

as $\epsilon \longrightarrow 0$ for each $T>0$. 


\section{The single quasilinear parabolic equation}

This section is devoted to problem (1.2) in the scalar case. We assume throughout that $f \in \mathcal{C}^{\infty}(\mathbb{R})$. For the time being, $\mu>0$ is considered a classical real number. We begin by recalling a Gronwall-type inequality which will be frequently needed in the sequel (for a proof, see [2, Lemma 2.2]).

Lemma 3.1: Let $w$ be a nonnegative, continuous function on $[0, \infty)$ and assume that

$$
w(t) \leq a+b \int_{0}^{t} \frac{w(s)}{\sqrt{t-s}} d s
$$

for some contants $a, b \geq 0$ and every $t \geq 0$. Then

$$
w(t) \leq a(1+2 b \sqrt{t}) \exp \left(\pi b^{2} t\right) .
$$

Next, we summarize the classical results concerning problem (1.2) which we need:

Lemma 3.2: Given $u_{0} \in \mathcal{C}_{b}^{\infty}(\mathbb{R})$, problem (1.2) has a unique solution $u \in \mathcal{C}_{b}^{\infty} \cap$ $L^{\infty}\left(\mathbb{R}_{+}^{2}\right)$. Moreover,

$$
\|u\|_{L^{\infty}\left(\boldsymbol{R}_{+}^{2}\right)} \leq\left\|u_{0}\right\|_{L^{\infty}(\boldsymbol{R})}
$$

Proof: Estimate (3.2) is just the maximum principle for classical solutions. The existence of a solution can be inferred as follows. Let

$$
E(x, t)=\frac{1}{2 \sqrt{\pi \mu t}} e^{-\frac{x^{2}}{1 \mu t}}
$$

be the fundamental solution of the heat operator. We rewrite (1.2). by means of Duhamel's principle as the integral equation

$$
\begin{aligned}
u(x, t)= & E(t) * u_{0}-\int_{0}^{t} E(s) * f(u)_{x}(x, t-s) d s \\
= & \frac{1}{\sqrt{\pi}} \int_{-\infty}^{\infty} e^{-v^{2}} u_{0}(x-2 \sqrt{\mu t} y) d y \\
& +\int_{0}^{t} \frac{1}{\sqrt{\pi \mu s}} \int_{-\infty}^{\infty} y e^{-y^{2}} f(u(x-2 \sqrt{\mu s} y, t-s)) d y d s
\end{aligned}
$$

where the derivation with respect to $x$ has been placed on the heat kernel by an integration by parts. The operator on the right-hand side of (3.3) is easily seen to determine a contraction in a suitable ball in the space $\mathcal{C}\left(\left[0, T_{k}\right]: \mathcal{C}_{b u}^{k}(\mathbb{R})\right)$ for every $k \in \mathbb{I}$. and sufficiently. small $T_{k}>0$, where $\mathcal{C}_{b u}^{k}(I R)$ denotes the space of functions with bounded and uniformly continuous derivatives up to order $k$. Thus a local solution $u$ exists. It is not difficult to see, say with $k=2$, that this function $u$ is a distribution solution to (1.2), 
from which the regularity of $u_{t}$ follows, so that $u$ is also'a classical solution. Now the maximum principle (3.2) applies, giving an a priori estimate entailing that the existence is global and producing a solution $u \in \mathcal{C}\left([0, \infty): \mathcal{C}_{b}^{\infty}(I R)\right)$. Its smoothness properties with respect to $t$ can be obtained by successively differentiating (1.2).

Finally, uniqueness follows by applying Lemma 3.1 in the situation of (3.3) 1

We now turn to generalized solutions to problem (1.2).

Theorem 3.3: Suppose that the function $f$ is slowly increasing at infinity, $\left|f^{\prime}\right|$ is bounded, and $\mu>0$. Then for any given $u_{0} \in \mathcal{G}_{g}(\mathbb{R})$, there exists a unique solution $u \in \mathcal{G}_{g}\left(\mathbb{R}_{+}^{2}\right)$ to problem (1.2).

Proof: We fix some representative $u_{0 e}$ of $u_{0}$, and consider the problem

$$
\left\{\begin{array}{l}
u_{\epsilon t}+f\left(u_{\epsilon}\right)_{x}=u_{\epsilon x x} \\
\left.u_{\epsilon}\right|_{t=0}=u_{0 \varepsilon}(x)
\end{array}\right.
$$

By Lemma 3.2, there is a unique solution $u_{c} \in \mathcal{C}_{\delta}^{\infty}\left(\mathbb{R}_{+}^{2}\right)$ of this problem. Using the fact that $u_{0 e} \in \mathcal{E}_{M, g}[R]$, the inequality (3.2) immediately implies that there is $N_{0} \in \mathbb{N}$ such that the solution $u_{e}$ satisfies

$$
\sup _{(x, t) \in R_{+}^{2}}\left|u_{c}(x, t)\right|=O\left(\epsilon^{-N_{0}}\right) \quad \text { as } \epsilon \longrightarrow 0 .
$$

Now we estimate $u_{e x}$. Replacing $u$ by $u_{\varepsilon}$ and $u_{0}$ by $u_{0 e}$ in (3.3), differentiating with respect to $x$ and taking the supremum gives

$$
\begin{aligned}
& \left\|u_{e x}(\cdot, t)\right\|_{L^{\infty}(\boldsymbol{R})} \\
& \quad \leq\left\|u_{0 e x}\right\|_{L^{\infty}(\boldsymbol{R})}+\frac{C}{\sqrt{\mu}}\left\|f^{\prime}\left(u_{e}\right)\right\|_{L^{\infty}} \int_{0}^{t_{0}} \frac{1}{\sqrt{t-s}}\left\|u_{e x}(\cdot, s)\right\|_{L^{\infty}(\boldsymbol{R})} d s .
\end{aligned}
$$

Employing Lemma 3.1, this inequality implies

$$
\begin{aligned}
& \left\|u_{e x}(\cdot, t)\right\|_{L^{\infty}(\boldsymbol{R})} \\
& \quad \leq\left\|u_{0 \epsilon x}\right\|_{L^{\infty}(R)}\left(1+\frac{2 C}{\sqrt{\mu}}\left\|f^{\prime}\left(u_{\varepsilon}\right)\right\|_{L^{\infty}} \sqrt{t}\right) \exp \left(\pi \frac{C^{2}}{\mu}\left\|f^{\prime}\left(u_{\epsilon}\right)\right\|_{L^{\infty}}^{2} t\right) .
\end{aligned}
$$

Since $\left|f^{\prime}\right|$ is bounded, (3.7) implies that, for any $T>0$, there is $N_{1} \in \dot{N}$ such that

$$
\sup _{(x, t) \in R \times[0, T]}\left|u_{e x}(x, t)\right|=O\left(\epsilon^{-N_{1}}\right) \quad \text { as } \epsilon \longrightarrow 0 .
$$

We can similarly prove that the same type of estimate is still valid for the higher derivatives of $u_{e}$ with respect to $x$, noting that the highest order derivative of $u$ always goes with the factor $f^{\prime}\left(u_{\varepsilon}\right)$. Concerning the $t$-derivatives and the mixed ones, using the differential equation (3.4) and by differentiation we can successively get a similar estimate. That is to say that $\left\{u_{c}\right\}_{c>0}$ belongs to $\mathcal{E}_{M, g}\left[\mathbb{R}_{+}^{2}\right]$; its class in $\mathcal{G}_{g}\left(\mathbb{R}_{+}^{2}\right)$ defines a generalized solution to problem (1.2). 
To prove uniqueness, suppose that $u_{1}, u_{2} \in \mathcal{G}_{g}\left(\mathbb{R}_{+}^{2}\right)$ are two solutions to problem (1.2). Then there are $N \in \mathcal{N}_{g}\left(\mathbb{R}_{+}^{2}\right)$ and $n \in \mathcal{N}_{g}(\mathbb{R})$ such that

$$
\left\{\begin{aligned}
\left(u_{1 \epsilon}-u_{2 \epsilon}\right)_{t}+f\left(u_{1 \epsilon}\right)_{x}-f\left(u_{2 \epsilon}\right)_{x} & =\mu\left(u_{1 \ell}-u_{2 \epsilon}\right)_{x x}+N_{\epsilon} \\
\left.\left(u_{1 \epsilon}-u_{2 \epsilon}\right)\right|_{t=0} & =n_{e}(x)
\end{aligned}\right.
$$

with $u_{1 \ell}, u_{2 \ell}$ being representatives of $u_{1}, u_{2}$, and $N_{\ell}, n_{\epsilon}$ defining $N, n$, respectively. It follows that the difference $\left(u_{1 \epsilon}-u_{2 \epsilon}\right)(x, t)$ equals

$$
\begin{aligned}
& \frac{1}{\sqrt{\pi}} \int_{-\infty}^{\infty} e^{-y^{2}} n_{\epsilon}(x-2 \sqrt{\mu t} y) d y \\
& \quad+\frac{1}{\sqrt{\pi}} \int_{0}^{t} \int_{-\infty}^{\infty} e^{-y^{2}} N_{\epsilon}(x-2 \sqrt{\mu s} y, t-s) d y d s \\
& \quad+\int_{0}^{t} \frac{1}{\sqrt{\pi \mu s}} \int_{-\infty}^{\infty} y e^{-y^{2}}\left[f\left(u_{1 \epsilon}(x-2 \sqrt{\mu s} y, t-s)\right)\right. \\
& \left.\quad-f\left(u_{2 \epsilon}(x-2 \sqrt{\mu s} y, t-s)\right)\right] d y d s
\end{aligned}
$$

which implies

$$
\begin{aligned}
\left\|\left(u_{1 e}-u_{2 e}\right)(\cdot, t)\right\|_{L^{\infty}(\boldsymbol{R})} \leq & \left\|n_{e}\right\|_{L^{\infty}(\boldsymbol{R})}+t\left\|N_{e}\right\|_{L^{\infty}(\boldsymbol{R} \times\{0, T])} \\
\ddots & +\frac{C}{\sqrt{\mu}}\left\|f^{\prime}\right\|_{L^{\infty}} \int_{0}^{t} \frac{1}{\sqrt{t-s}}\left\|\left(u_{1 e}-u_{2 e}\right)(\cdot, s)\right\|_{L^{\infty}(R)} d s .
\end{aligned}
$$

Again using (3.1) it follows that

$$
\begin{aligned}
& \left\|\left(u_{1 \epsilon}-u_{2 \epsilon}\right)(\cdot, t)\right\|_{L^{\infty}(\boldsymbol{R})} \\
& \quad \leq\left(\left\|n_{e}\right\|_{L^{\infty}(\boldsymbol{R})}+t\left\|N_{\epsilon}\right\|_{L^{\infty}(\boldsymbol{R} \times[0, T])}\right)\left(1+\frac{2 C}{\sqrt{\mu}}\left\|f^{\prime}\right\|_{L^{\infty}} \sqrt{t}\right) \exp \left(\frac{\pi C^{2}}{\mu}\left\|f^{\prime}\right\|_{L^{\infty}}^{2} t\right)
\end{aligned}
$$

which means that

$$
\sup _{(x, t) \in \mathbb{R} \times[0, T]}\left|\left(u_{1 \ell}-u_{2 \epsilon}\right)(x, t)\right|=\dot{O}\left(\epsilon^{M}\right) \quad \text { as } \epsilon \longrightarrow 0
$$

for any $T>0$ and $M \in \mathbb{N}$ since $n \in \mathcal{N}_{g}(\mathbb{R}), N \in \mathcal{N}_{g}\left(\mathbb{R}_{+}^{2}\right)$ and $\left|f^{\prime}\right|$ is bounded.

For the derivatives of $u_{1 \epsilon}-u_{2 \varepsilon}$ with respect to $(x, t)$ we can similarly prove that the same type of estimate as (3.10) is still valid by using the arguments of the existence part. Hence, $u_{1}-u_{2} \in \mathcal{N}_{g}\left(\mathbb{R}_{+}^{2}\right)$ which completes our proof

Theorem 3.4: 'Suppose that $f$ is slowly increasing at infinity and $f^{\prime}$ is of order $r$.

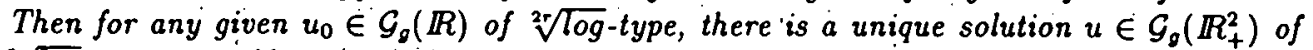

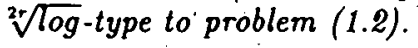

-Proof: Under the above assumtions, we have that the representative $u_{0 e}$ of $u_{0}$ satisfies

$$
\left\|u_{0 e}\right\|_{L^{\infty}(R)} \leq C \sqrt[2 r]{|\log \epsilon|}
$$


with $C>0$ being a constant. Using (3.2) it follows that the solution $u_{\varepsilon}$ to (3.4) satisfies

$$
\left\|u_{\epsilon}\right\|_{L^{\infty}\left(\boldsymbol{R}_{+}^{2}\right)} \leq C \sqrt[2 r]{|\log \epsilon|}
$$

which implies that

$$
\left\|f^{\prime}\left(u_{\varepsilon}\right)\right\|_{L^{\infty}\left(\boldsymbol{R}_{+}^{2}\right)} \leq C_{1} \sqrt{|\log \epsilon|}
$$

with $C_{1}>0$ being a constant. The inequality (3.7) gives rise to the assertion that the solution to (3.4) still satisfies (3.8). For the higher derivatives of $u_{\ell}$ with respect to $(x, t)$ we can easily establish the same type of estimate by using the method of Theorem 3.3 and (3.13). That is to say that the class of $\left\{u_{c}\right\}_{c>0} \in \mathcal{G}_{g}\left(\mathbb{R}_{+}^{2}\right)$, which is of $\sqrt[2 r]{\log }$-type, is a solution of the problem (1.2).

Now we prove uniqueness. If $u_{1}, u_{2} \in \mathcal{G}_{g}\left(\mathbb{R}_{+}^{2}\right)$ are two solutions to problem (1.2) with representatives $u_{1 e}$ and $u_{2 e}$ both being of $\sqrt[2 r]{l o g}$-type, then there are $N \in \mathcal{N}_{g}\left(\mathbb{R}_{+}^{2}\right)$ and $n \in \mathcal{N}_{g}(\mathbb{R})$ such that

$$
\left\{\begin{aligned}
\left(u_{1 \ell}-u_{2 \ell}\right)_{t}+f\left(u_{1 \epsilon}\right)_{x}-f\left(u_{2 \ell}\right)_{x} & =\mu\left(u_{1 \epsilon}-u_{2 \epsilon}\right)_{x x}+N_{\epsilon} \\
\left.\left(u_{1 \ell}-u_{2 \ell}\right)\right|_{t=0} & =n_{\epsilon}(x)
\end{aligned}\right.
$$

where $N_{e}$ and $n_{e}$ are the defining sequences of $N$ and $n$, respectively. Since $u_{1 e}$ and $u_{2 \varepsilon}$

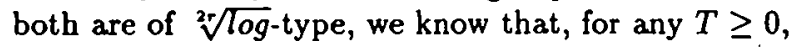

$$
\therefore \sup _{(x, t) \in \operatorname{R\times }[0, T]}\left|\int_{0}^{1} f^{\prime}\left(\theta u_{1 e}(x, t)+(1-\theta) u_{2 \ell}(x, t)\right) d \theta\right| \leq C_{T} \sqrt{|\log \epsilon|}
$$

with $C_{T}>0$ being a constant. Therefore, we can easily obtain that the class of $\left\{u_{1 e}-\right.$ $\left.u_{2 \varepsilon}\right\}_{\text {e>0 }}$ belongs to the set $\mathcal{N}_{g}\left(\mathbb{R}_{+}^{2}\right)$ by using the same method as in the uniqueness part of Theorem 3.3

Formally setting $r=\infty$ in Theorem 3.4 we get the following stronger result for initial data of bounded type.

Corollary 3.5: Suppose $f$ is slowly increasing at infinity. Then for any given $u_{0} \in$ $\mathcal{G}_{g}(\mathbb{R})$ of bounded type; there is a unique solution $u \in \mathcal{G}_{g}\left(\mathbb{R}_{+}^{2}\right)$ of bounded type to problem (1.2).

Proof: By assumption, $\left\|u_{0 e}\right\|_{L^{\infty}(R)} \leq M$ for some $M$ uniformly in $\epsilon>0$; by the maximum principle (3.2), $\left|u_{e}\right|$ is majorized by $M$ as well, so $\left|f^{\prime}\left(u_{e}\right)\right|$ is uniformly bounded independently of $\epsilon>0$. The same arguments as above go through

Remark 3.6: Concerning the generalized solutions constructed in Theorem3.3, Theorem 3.4 and Corollary 3.5, we can immediately assert the folluwing consistency property: If the initial data $u_{0}$ actually belong to $\mathcal{C}_{b}^{\infty}(I R)$, then the generalized solution coincides with the classical solution in $\mathcal{G}_{g}\left(\mathbb{R}_{+}^{2}\right)$. Indeed, as noted after $(2.2), u_{0}$ may itself serve as a representative for $\iota_{\rho}\left(u_{0}\right)$, so the classical solution in $\mathcal{C}_{\bar{b}}^{\infty}\left(\overline{I R_{+}^{2}}\right)$ is a representative for the generalized solution. The relation with the classical solution when $u_{0} \in L^{\infty}(\mathbb{R})$ will be discussed below. 
We now study problem (1.2) when $\mu$ is allowed to be a generalized constant. In order not to complicate matters we just give a typical result, corresponding to Corollary 3.5 .

Theorem 3.7: Suppose that $\mu$ is a generalized positive number such that $1 / \mu$ is of $\log$-type. Let $f$ be slowly increasing at infinity and let $u_{0} \in \mathcal{G}_{g}(\mathbb{R})$ be of bounded type. Then there is a unique solution $u \in \mathcal{G}_{g}\left(\mathbb{R}_{+}^{2}\right)$ of bounded type to problem (1.2).

Proof: As in Corollary 3.5, we have that $\left|f^{\prime}\left(u_{\epsilon}\right)\right|$ is bounded independently of $\epsilon>0$. The rest follows as in the previous theorems, noting that $\mu_{e}^{-1}=O(\log (1 / \epsilon))$ in the inequalities of type (3.7)

We remark that the hypothesis requires $\mu_{c}$ to converge to zero rather slowly, if at all. Various improved estimates can be obtained if $f$ is homogeneous of degree $p$ : Then the change of variable $v_{e}(x, t)=\mu_{e}^{\frac{1}{2-2 p}} u_{e}\left(\sqrt{\mu_{c}} x, t\right)$ reduces problem (1.2) to the case with $\mu_{c} \equiv 1$, thus the previous estimates apply, and the factor $\mu_{e}^{1 /(2-2 p)}$ determines the type of $v_{e}$.

We now undertake a comparison of the generalized solutions obtained so far with classical solutions, when the latter exist. First some preparatory observations: Lax [7; Section 5] introduced the following pseudo-norm, defined on locally integrable functions $g$ on $\mathbb{R}$, but possibly infinite:

$$
|g|_{0}=\sup _{x \in \mathbb{R}}\left|\int_{0}^{x} g(\xi) d \xi\right|
$$

and showed that if $u_{1}$ and $u_{2}$ are two classical solutions to problem (1.2) with initial data $u_{01}$ and $u_{02}$, then $\left|\left(u_{1}-u_{2}\right)(\cdot, t)\right|_{*} \leq 2\left|u_{01}-u_{02}\right|_{*}$ for any $t \geq 0$. We also note that if $\left|g_{e}\right|_{.} \longrightarrow 0$ as $\epsilon \longrightarrow 0$, then $g_{e} \longrightarrow 0$ in $\mathcal{D}^{\prime}(\mathbb{R})$. Further, letting $u_{0} \in L^{\infty}(\mathbb{R})$. and $\rho_{\mathrm{e}}$ as in (2.1), (2.2), it holds that $\left|u_{0}-u_{0} * \rho_{\varepsilon}\right|_{*} \longrightarrow 0$ as $\epsilon \longrightarrow 0$. For proofs of these assertions we refer to [2, Section 3$]$.

We now take initial data $u_{0} \in L^{\infty}(\mathbb{R})$ and let $\mu$ be a positive number or a generalized positive number such that $1 / \mu$ is of log-type. According to Corollary 3.5 or Theorem 3.7, the problem

$$
\left\{\begin{array}{l}
u_{t}+f(u)_{x}=\mu u_{x x} \\
\left.u\right|_{t=0}=\iota_{p}\left(u_{0}\right)
\end{array} .\right.
$$

has a unique solution $u \in \mathcal{G}_{g}\left(\mathbb{R}_{+}^{2}\right)$ of bounded type. Let $\left\{\mu_{e}\right\}_{e>0}$. be a representative of $\mu$ and consider the problem

$$
\left\{\begin{array}{l}
v_{e t}+\cdot f\left(v_{e}\right)_{x}=\mu_{e} v_{e x x} \\
\left.v_{e}\right|_{t=0}=u_{0}(x)
\end{array}\right.
$$

In order to avoid additional regularity considerations, we just assume that $u_{0} \in \mathcal{C}_{b u}^{2}(\mathbb{R})$. According to the arguments sketched at the beginning of this section, problem (3.15) has a unique classical solution $v_{c}$. Finally, let $\left\{u_{c}\right\}_{c>0}$ be the representative of the generalized solution to problem (3.14) constructed in Theorem 3.7 and tiie preceding results, i.e. $u_{e}$ solves the problem

$$
\left\{\begin{array}{l}
u_{t t}+f\left(u_{c}\right)_{x}=\mu_{e} u_{e x x} \\
\left.u_{e}\right|_{t=0}=u_{0} * \dot{\rho}_{c}(x) .
\end{array}\right.
$$


Lemma 3.8: The difference $u_{e}-v_{e}$ converges to zero in $\mathcal{D}^{\prime}\left(I_{+}^{2}\right)$ as $\epsilon \longrightarrow 0$.

Proof: According to the observations above, $\sup _{t \geq 0}\left|\left(v_{\mathrm{e}}-u_{\mathrm{c}}\right)(\cdot, t)\right|_{*} \leq 2\left|u_{0}-u_{0} * \rho_{\mathrm{c}}\right|_{*}$ tends to zero with $\epsilon$, and this entails the distributional convergence of $u_{\epsilon}-v_{\epsilon}$ to zero

Proposition 3.9: Let $f$ be slowly increasing at infinity, $\mu$ a positive real number and $u_{0} \in \mathcal{C}_{b u}^{2}(I R)$. Then the generalized solution $u \in \mathcal{G}_{g}\left(\mathbb{R}_{+}^{2}\right)$ to problem (3.14) is associated with the classical solution $v$ to the problem

$$
v_{t}+f(v)_{x}=\mu v_{x x},\left.\quad v\right|_{t=0}=u_{0}(x)
$$

Proof: Since $\mu$ is a classical real number, we may set $\mu_{c} \equiv \mu$ in (3.15) and (3.16). Thus $v_{\mathrm{e}} \equiv v$ in (3.15), and the assertion follows immediately from Lemma 3.8

Proposition 3.10: Let $f$ be slowly increasing at infinity, $\mu$ a generalized positive number such that $1 / \mu$ is of log-type, $u_{0} \in \mathcal{C}_{b u}^{2} \cap L^{1}(\mathbb{R})$. If $\mu \approx 0$, then the generalized solution $u \in \mathcal{G}_{g}\left(\mathbb{R}_{+}^{2}\right)$ to problem (3.14) is associated with the weak entropic solution $w \in L^{\infty}\left(\mathbb{R}_{+}^{2}\right)$ to the conservation law

$$
w_{t}+f(w)_{x} \doteq 0,\left.\quad w\right|_{i=0}=u_{0}(x)
$$

Proof: With $v_{e}$ and $u_{e}$ as in (3.15) and (3.16), we have $u_{e}-v_{e} \longrightarrow 0$ in $\mathcal{D}^{\prime}\left(\mathbb{R}_{+}^{2}\right)$. On the other hand, the solutions $v_{e}$ are known to converge in $L_{l o c}^{1}\left(I_{+}^{2}\right)$ to the weak entropic solution $w$ as $\mu_{c} \longrightarrow 0$ (see, e.g., DiPerna [6: Theorem 4.3]). Thus $u_{e} \longrightarrow w$ in $\mathcal{D}^{\prime}\left(\mathbb{R}_{+}^{2}\right)$ as $\epsilon \longrightarrow 0$, so $u \approx w$ प

We point out that in the situation of Proposition 3.10, the generalized solution $u \in$ $\mathcal{G}_{g}\left(\mathbb{R}_{+}^{2}\right)$ is also a solution to problem (1.3), more precisely,

$$
u_{t}+f(u)_{x} \approx 0,\left.\quad u\right|_{i=0} \approx u_{0} .
$$

This follows from the fact that $u$ is of bounded type and $\mu \approx 0$, so $\mu u_{x x} \approx 0$.

\section{Systems of conservation laws}

We turn to the case where (1.2) is a system of equations, that is, $u=\left(u_{1}, \ldots, u_{n}\right)$ and $f=\left(f_{1}, \ldots, f_{n}\right)$. The constant $\mu$ is taken the same in each component. The integral equation (3.3) now turns into a system of integral equations, valid for each row with $u$, $u_{0}, f(u)$ replaced by $u_{i}, u_{0 i}, f_{i}(u)(i=1, \ldots, n)$. The existence of a local classical solution is established easily by the same arguments as in Section 3. However, as opposed to the scalar case, no maximum principle is available in general. Thus further ingredients are needed to enable the extension of the local solution to a global one. We begin by discussing a simple situation where an a priori estimate is nevertheless valid, namely when $\left|f^{\prime}\right|$ is globally bounded. In this situation one can write

$$
f(u)=\int_{0}^{1} f^{\prime}(\sigma u) d \sigma \cdot u+f(0)
$$


and apply the Growall-type inequality of Lemma 3.1 to obtain an a priori estimate of the form

$$
\|u\|_{L^{\infty}(\boldsymbol{R} \times(0, T))} \leq C\left(T,\left\|f^{\prime}\right\|_{L^{\infty}\left(\boldsymbol{R}^{n}\right)}\right) .
$$

On the one hand, this implies global existence of a classical solution, as in the scalar case. On the other hand, concerning generalized solutions in $\mathcal{G}_{g}\left(\mathbb{R}_{+}^{2}\right)$, Theorem 3.3 carries over literally.

Proposition 4.1: Suppose that $f: \mathbb{R}^{n} \longrightarrow \mathbb{R}^{n}$ is slowly increasing at infinity and $\left|f^{\prime}\right|$ is bounded, $\mu>0$. Then for any $u_{0}=\left(u_{01}, \ldots, \dot{u}_{0 n}\right) \dot{\in}\left(\mathcal{G}_{g}(R)\right)^{n}$, there is a unique solution $u=\left(u_{1}, \ldots, u_{n}\right) \in\left(\mathcal{G}_{g}\left(R_{+}^{2}\right)\right)^{n}$ to system (1.2).

A more interesting situation arises when system (1.2) admits an invariant region on which $\left|f^{\prime}\right|$ is bounded. A subset $\Sigma$ of $\mathbb{R}^{n}$ is called an invariant region for (1.2) if, given any $T>0$ and a classical solution $u$ to $(1.2)$ on $\mathbb{R} \times[0, T), u_{0}(x) \in \Sigma$ for all $x \in \mathbb{R}$ implies $u(x, t) \in \dot{\Sigma}$ for all $(x, t) \in \mathbb{R} \times[0, T)$.

Definition 4.2: An element $u \in\left(\mathcal{G}_{g}\left(\mathbb{R}_{+}^{2}\right)\right)^{n}$ is called of type $\Sigma$, if for any representative $\left\{u_{e}\right\}_{c>0}$ there is $\epsilon_{0}>0$ so that $u_{c}(x, t) \in \Sigma$ for all $x \in \mathbb{R}, t>0$, and $\epsilon<\epsilon_{0}$. A similar definition applies to $u_{0} \in\left(\mathcal{G}_{g}(\mathbb{R})\right)^{n}$.

Theorem 4.3: Suppose that $f: \mathbb{R}^{n} \longrightarrow \mathbb{R}^{n}$ is slowly increasing at infinity, $\mu>0$, and system (1.2) admits a convex invariant region $\Sigma$ on which $\left|f^{\prime}\right|$ is bounded. Then for any $u_{0}=\left(u_{01}, \ldots, u_{0 n}\right) \in\left(\mathcal{G}_{g}(\mathbb{R})\right)^{n}$ of type $\Sigma$, there is a unique solution $u=\left(u_{1}, \ldots, u_{n}\right) \in$ $\left(\mathcal{G}_{g}\left(\mathbb{R}_{+}^{2}\right)\right)^{n}$ of type $\Sigma$ to system (1.2).

Proof: Fix some point $z \dot{\epsilon} \Sigma$. Then we have for every $u \in \Sigma$ that

$$
\begin{aligned}
|f(u)| & =\left|\int_{0}^{1} f^{\prime}(\sigma u+(1-\sigma) z) d \sigma \cdot(u-z)+f(z)\right| \\
& \leq \sup _{\eta \in \Sigma}\left|f^{\prime}(\dot{\eta})\right|(|u|+|z|)+|f(z)| .
\end{aligned}
$$

Inserting this in the system of integral equations (3.3), applying Lemma 3.1 and observing that $\Sigma$ is an invariant region gives an a priori estimate. Consequently, classical solutions exist globally, provided the initial data take values in $\Sigma$. Uniqueness of classical solutions with values in $\Sigma$ is obtained by applying a similar argument to $f(u)-f(\bar{u})$.

Next, let $u_{0} \in\left(\mathcal{G}_{g}(\mathbb{R})\right)^{n}$ and take a representative $\left\{u_{0_{e}}\right\}_{e>0}$. According to the consideration above, there corresponds a unique classical solution $u_{e} \in \mathcal{C}_{b}^{\infty}\left(\mathbb{I R}_{+}^{2}\right)$ with $u_{e}(x, t) \in \Sigma$ for all $x \in \mathbb{R}, t>0$. Substituting $u_{e}$ in the place of $u$ in (4.1) and inserting this in the integral equations, produces for every $T>0$ the estimate $\left\|u_{c}\right\|_{L^{\infty}(R \times(0, T))}=O\left(\epsilon^{-N}\right)$ for some $N \in \mathbb{N}$. The estimate on $u_{c \dot{x}}$ follows from (3.6), (3.7) and the fact that $u_{e} \in \Sigma$. Higher order derivatives are estimated inductively as in Theorem 3.3. Uniqueness of solutions in $\mathcal{G}_{g}\left(\mathbb{R}_{+}^{2}\right)$ of type $\Sigma$ is proved along the same lines, noting that $\sigma u_{c}+(1-\sigma) \bar{u}_{e}$ belongs to $\Sigma$ for $0 \leq \sigma \leq 1$, if $u_{c}$ and $\bar{u}_{c}$ belong to $\Sigma$

Remark 4.4: An extension of Theorem 4.3 to include the case where $\mu$ is a generalized positive number such that $1 / \mu$ is of log-type is similarly valid, provided $\Sigma$ is an invariant region for all $\mu_{c}$ defining the generalized constant $\mu$. We leave the details to the reader. 
Example 4.5 (The p-system of gas dynamics with artificial viscosity): This is system (1.4) with $p(v)=k v^{-\gamma}, k>0$ and $\gamma>1$. According to Smoller [9, pp. $211-212$ ] (see also Chueh, Conley and Smoller [3]), an invariant region is given in terms of the Riemann invariants

$$
r(v, u)=u-2 \sqrt{k \gamma}(1-\gamma)^{-1} v^{(1-\gamma) / 2}, \quad s(v, u)=u+2 \sqrt{k \gamma}(1-\gamma)^{-1} v^{(1-\gamma) / 2}
$$

by $\Sigma=\left\{(v, u) \in \mathbb{R}^{2}: r \leq r_{0}, s \geq s_{0}\right\}$. Taking in particular $r_{0}=-s_{0}>0,(v, u) \in \Sigma$ implies that $|u| \leq r_{0}$ and $v \geq\left((4 k \gamma)^{-1}(\gamma-1) r_{0}\right)^{2 /(1-\gamma)} \longrightarrow 0$ as $r_{0} \longrightarrow \infty$. For fixed $\delta>0, \Sigma$ eventually contains the region $|u| \leq 1 / \delta, v \geq \delta$. In addition,

$$
f^{\prime}(v, u)=\left(\begin{array}{cc}
0 & 1 \\
p^{\prime}(v) & 0
\end{array}\right)
$$

is clearly bounded on $\Sigma$ for fixed $r_{0}$. Therefore, if the initial data $\left(v_{0}, u_{0}\right) \in\left(\mathcal{G}_{g}(\mathbb{R})\right)^{2}$ are such that $u_{0}$ is of bounded type and $v_{0}$ has a representative satisfying $v_{0 \varepsilon} \geq \delta>0$ for all $\epsilon$, then Theorem 4.3 applies and so there is a solution $(v, u) \in\left(\mathcal{G}_{g}\left(\mathbb{R}_{+}^{2}\right)\right)^{2}$, which is of corresponding type $\Sigma$ and unique with this property.

Acknowledgement: The second author would like to express his gratitude to the Institut für Mathematik und Geometrie, Universität Innsbruck for the hospitality while he stayed there from April 1991 to April 1992, and to the Austrian Academic Exchange Service for the financial support.

\section{References}

[1] Biagioni, H.A.: A Nonlinear Theory of Generalized Functions. Lect. Notes Math. 1421 (1990), 1 - 214.

[2] Biagioni, H.A., and M. Oberguggenberger: Generalized solutions to Burgers' equation. J. Diff. Eqs. 97 (1992), 263 - 287.

[3] Chueh, K.N., Conley, C.C., and J.A. Smoller: Positively invariant regions for systems of nonlinear diffusion equations. Indiana Univ. Math. J. 26 (1977), 373 - 392.

[4] Colombeau, J.F.: New Generalized Functions and Multiplication of Distributions (North - Holland Math. Studies: Vol. 84). Amsterdam: North - Holland 1984.

[5] Cólom Beau, J. F.: Elementary Introduction to New Generalized Functions(North-Holland Math. Studies: Vol. 113). Amsterdam: North - Holland 1985.

[6] DiPerna, R.J.: Measure-valued solutions to conservation laws. Arch. Rat. Mech. Anal. 88 (1985), $223-270$.

[7]. LAX, P.D.: Weak solutions of nonlinear hyperbolic equations and their numerical computation. Comm. Pure Appl. Math. 7 (1954), 159 - 193.

[8] Oberguggenberger, M.: Multiplication of Distributions and Applications to Partial Differential Equations (Pitman Research Notes Math.: Vol. 259). Harlow: Longman 1992.

[9] Smoller, J.A.: Shock Waves and Reaction-Diffusion Equations (Grundlehren Math. Wiss.: Vol. 258). New York: Springer - Verlag 1983.

Received 05.04.1993 